\title{
Bayesian methods in structural bioinformatics
}

\author{
Hamelryck, Thomas Wim; Mardia, Kanti; Ferkinghoff-Borg, Jesper
}

Link to article, DOI:

10.1007/978-3-642-27225-7

Publication date:

2012

Document Version

Early version, also known as pre-print

Link back to DTU Orbit

Citation (APA):

Hamelryck, T. W., Mardia, K., \& Ferkinghoff-Borg, J. (Eds.) (2012). Bayesian methods in structural bioinformatics. Springer. Statistics for Biology and Health, Springer https://doi.org/10.1007/978-3-642-27225-7

\section{General rights}

Copyright and moral rights for the publications made accessible in the public portal are retained by the authors and/or other copyright owners and it is a condition of accessing publications that users recognise and abide by the legal requirements associated with these rights.

- Users may download and print one copy of any publication from the public portal for the purpose of private study or research.

- You may not further distribute the material or use it for any profit-making activity or commercial gain

- You may freely distribute the URL identifying the publication in the public portal

If you believe that this document breaches copyright please contact us providing details, and we will remove access to the work immediately and investigate your claim 


\section{Statistics for Biology and Health}

Series Editors:

M. Gail

K. Krickeberg

J. Samet

A. Tsiatis

W. Wong

For other titles published in this series, go to

http://www.springer.com/series/2848 

Thomas Hamelryck - Kanti Mardia Jesper Ferkinghoff-Borg Editors

\section{Bayesian Methods in Structural Bioinformatics}

望 Springer 


\section{Editors}

Thomas Hamelryck

University of Copenhagen

Department of Biology

Bioinformatics Centre

Copenhagen

Denmark

\section{Kanti Mardia}

University of Leeds

School of Mathematics

Department of Statistics

Leeds

United Kingdom
Jesper Ferkinghoff-Borg

Technical University of Denmark

Department of Electrical Engineering

Lyngby

Denmark

Statistics for Biology and Health Series Editors

\author{
M. Gail \\ National Cancer Institute \\ Bethesda, MD \\ USA
}

Klaus Krickeberg

Le Châtelet

Manglieu

France

Jonathan M. Samet

Department of Preventive Medicine

Keck School of Medicine

University of Southern California

Los Angeles, CA

USA

\author{
A. Tsiatis \\ Department of Statistics \\ North Carolina State University \\ Raleigh, NC \\ USA
}

W. Wong

Department of Statistics

Stanford University

Stanford, CA

USA

ISSN 1431-8776

ISBN 978-3-642-27224-0

DOI 10.1007/978-3-642-27225-7

e-ISBN 978-3-642-27225-7

Springer Heidelberg Dordrecht London New York

Library of Congress Control Number: 2012933773

(c) Springer-Verlag Berlin Heidelberg 2012

This work is subject to copyright. All rights are reserved, whether the whole or part of the material is concerned, specifically the rights of translation, reprinting, reuse of illustrations, recitation, broadcasting, reproduction on microfilm or in any other way, and storage in data banks. Duplication of this publication or parts thereof is permitted only under the provisions of the German Copyright Law of September 9, 1965, in its current version, and permission for use must always be obtained from Springer. Violations are liable to prosecution under the German Copyright Law.

The use of general descriptive names, registered names, trademarks, etc. in this publication does not imply, even in the absence of a specific statement, that such names are exempt from the relevant protective laws and regulations and therefore free for general use.

Printed on acid-free paper

Springer is part of Springer Science+Business Media (www.springer.com) 
Thomas Hamelryck dedicates his contributions to his mother Gib, and to the memory of his father Luc (1942-2011).

Kanti Mardia dedicates his contributions to his grandson Sashin Raghunath. 



\section{Foreword}

The publication of this ground-breaking and thought-provoking book in a prestigious Springer series will be a source of particular pleasure and of stimulus for all scientists who have used Bayesian methods in their own specialized area of Bioinformatics, and of excitement for those who have wanted to understand them and learn how to use them but have never dared ask.

I met the lead author, Dr. Hamelryck, at the start of his career, when, as part of his $\mathrm{PhD}$ in Protein Crystallography, he determined and proceeded to analyze in great detail the 3D structures of several tight protein-carbohydrate complexes. His attention was drawn to Bayesian and related statistical methods by the profound impact they were having at the time on the two workhorses of macromolecular crystallography, namely experimental phasing and structure refinement. In both cases, recourse to the key Bayesian concept of marginalisation with respect to the phases (treated as nuisance parameters) freed those techniques from the limitations inherent in their old, least-squares based implementations, and gave access, through a shift to a maximum-likelihood approach, to much higher quality, much less biased electrondensity maps and atomic models. Preferring the world of computational methods to the biochemist's bench, Dr. Hamelryck went for a post-doc in Copenhagen, in the laboratory where he subsequently developed a highly productive structural extension to the already prominent Bioinformatics Department headed by Prof. Anders Krogh. The contents of this book include a representative sample of the topics on which he and his collaborators have focussed their efforts since that time.

The use of advanced statistical methods in Bioinformatics is of course well established, with books such as Biological Sequence Analysis - Probabilistic Models of Proteins and Nucleic Acids by Durbin, Eddy, Krogh and Mitchison setting a very high standard of breadth, rigour and clarity in the exposition of the mathematical techniques involved and in the description of their implementations. The present book, however, is the first of its kind in directing the arsenal of Bayesian methods and their advanced computational tools towards the analysis, simulation and prediction of macromolecular (especially protein) structure in three dimensions. 
This is an extremely ambitious goal, as this area comprises no less than the Protein Folding Problem, arguably the most fundamental riddle in the whole of the Life Sciences.

The book begins with an up-to-date coverage, in Part I, of the concepts of Bayesian statistics and of the versatile computational tools that have been developed to make their use possible in practice. A particular highlight of the book is found in Part II, which presents a review of the time-honored use of knowledge-based potentials obtained by data mining, along with a critical reassessment of their exact relationship with potentials of mean force in statistical physics. Its conclusion is a highly original and welcome contribution to the solution of this long-standing problem. Another highlight is the combined use in Part V of the type of directional statistics that can be compiled by the methods of Part IV with the technology of Bayesian networks to produce very efficient methods for sampling the space of plausible conformations of protein and RNA molecules.

It is fitting that the book should return in its final Part VI to the interface between Bayesian methods of learning the structural regularities of macromolecules from known 3D structures on the one hand, and experimental techniques for determining new 3D structures on the other. As is well known, most of these techniques (with the exception perhaps of ultra-high resolution X-ray crystallography with plentiful sources of experimental phase information) need to be supplemented with some degree of low-level a priori knowledge about bond lengths and bond angles to enforce sensible stereochemistry in the resulting atomic models. The Bayesian picture turns this conventional approach on its head, making the process look instead like structure prediction assisted by X-ray (or NMR) data, with the measurements delivered by each experimental technique providing the likelihood factor to supplement the prior probability supplied by previous learning, in order to cast the final result of this inference into the form of a posterior distribution over an ensemble of possible structures. It is only by viewing structure determination in this overtly Bayesian framework that the classical problems of model bias and map overinterpretation can be avoided; and indeed crystallographers, for example, are still far from having made full use of the possibilities described here. Dr. Hamelryck is thus likely to have paved the way for future improvements in his original field of research, in spite of having since worked in a related but distant area, using tools first developed and applied to address problems in medical diagnosis. This instance of the interconnectedness of different branches of science through their methods, and this entire book, provide a splendid illustration of the power of mathematical approaches as the ultimate form of re-useable thought, and of Bayesian methods in particular as a repository of re-useable forms of scientific reasoning.

I am confident that the publication of this book will act as a rallying call for numerous investigators using specific subsets of these methods in various areas of Bioinformatics, who will feel encouraged to connect their own work to the viewpoints and computational tools presented here. It can therefore be expected that this will lead to a succession of enlarged new editions in the future. Last but not 
least, this book should act as a magnet in attracting young researchers to learn these advanced and broadly adaptable techniques, and to apply them across other fields of science as well as to furthering the subject matter of the book itself.

Global Phasing Ltd.,

Cambridge, UK

Gerard Bricogne 



\section{Preface}

The protein folding problem is the loose denominator for an amalgam of closely related problems that include protein structure prediction, protein design, the simulation of the protein folding process and the docking of small molecules and biomolecules. Despite some, in our view, overly optimistic claims, ${ }^{1}$ the development of an insightful, well-justified computational model that routinely addresses these problems is one of the main open problems in biology, and in science in general, today [461]. Although there is no doubt that tremendous progress has been made in the conceptual and factual understanding of how proteins fold, it has been extraordinary difficult to translate this understanding into corresponding algorithms and predictions. Ironically, the introduction of CASP ${ }^{2}$ [400], which essentially evaluates the current state of affairs every two years, has perhaps lead to a community that is more focussed on pragmatically fine-tuning existing methods than on conceptual innovation.

In the opinion of the editors, the field of structural bioinformatics would benefit enormously from the use of well-justified machine learning methods and probabilistic models that treat protein structure in atomic detail. In the last 5 years, many classic problems in structural bioinformatics have now come within the scope of such methods. For example, conformational sampling, which up to now typically involved approximating the conformational space using a finite set of main chain fragments and side chain rotamers, can now be performed in continuous space using graphical models and directional statistics; protein structures can now be compared and superimposed in a statistically valid way; from experimental data, Bayesian methods can now provide protein ensembles that reflect the statistical uncertainty. All of these recent innovations are touched upon in the book, together with some more cutting edge developments that yet have to prove the extent of their merits.

\footnotetext{
${ }^{1}$ See for example Problem solved* (*sort of) in the news section of the August 8th, 2008 issue of Science, and the critical reply it elicited.

${ }^{2}$ CASP stands for "Critical Assessment of protein Structure Prediction".
} 
A comprehensive treatment of probabilistic methods in structural bioinformatics is, at first sight, something that would require several weighty book volumes. However, upon closer scrutiny, it becomes clear that the use of well-justified probabilistic methods in structural bioinformatics is currently in its infancy, and that their potential is enormous. Many knowledge based methods that claim to be firmly rooted in probability theory or statistical physics are at best heuristic methods that are often only partly understood. A classic example are the so called potentials of mean force that make use of pairwise distances in proteins. The validity and scope of these potentials have been topics of hot debate for over twenty years. Indeed, methods that both consider biomolecular structure in atomic detail and have a sound probabilistic justification are currently far and between.

In this book, we therefore focus on methods that have two important features in common. First, the focus lies on methods that are well justified from a probabilistic point of view, even if they are approximative. Quite a few chapters make use of point estimates, such as empirical Bayes, maximum likelihood or even moment estimates. However, in all cases, these are used as valid approximations of a true Bayesian treatment. Second, the methods deal with biomolecular structure in atomic detail. In that respect, classic applications of probabilistic reasoning in structural bioinformatics such as secondary structure prediction fall outside the scope of the book.

This book should be of use to both novices and experts, though we do assume knowledge of structural biology. Introductory chapters on Bayesian methods and Markov chain Monte Carlo methods, which play a key role in Bayesian inference, provide methodological background. These chapters will also be useful to experts in structural bioinformatics that are perhaps not so versed in probabilistic modelling. The remaining parts address various timely topics in structural bioinformatics; we give a short overview.

As mentioned before, the first two chapters provide the foundations. In the first chapter, Hamelryck gives a high level overview of the Bayesian interpretation of probability. The chapter also touches upon relevant topics in information theory and statistical mechanics, and briefly discusses graphical models. Despite the fact that Bayesian methods are now firmly established in statistics, engineering and science in general, most university courses on statistics in these disciplines still uniquely focus on frequentist statistics. The underlying reasons are clearly beyond the usual academic inertia, and can probably be identified with two main perceived problems [51]: Bayesian statistics is unjustly seen as inherently subjective and thus unsuitable for scientific research, and the difficulty of integrating the Bayesian view with the - for many applications still dominating - frequentist paradigm. In addition, many frequentist methods can be seen as perfectly valid approximations to Bayesian methods, thereby removing the apparent need for a paradigm shift. Therefore, we believe that the introductory chapter on Bayesian statistics is quite appropriate. Ferkinghoff-Borg provides an overview of Markov chain Monte Carlo methods. These sampling methods are vital for Bayesian inference, especially when it involves the exploration of the conformational space of proteins. Together, these two chapters should provide a decent start for anybody with some knowledge of 
structural bioinformatics, but a lacking background in Bayesian statistics. Both chapters focus on concepts rather than mathematical rigor and provide ample references for deeper study. However, these two chapters provide the bedrock on which the rest of the book is founded.

The second part addresses the estimation of so-called knowledge based potentials from data. Recently, dramatic progress has been made in the understanding of the statistics behind these potentials, which were previously justified using rather ad hoc physical arguments. The chapter by Borg et al. discusses knowledge based potentials from a physical viewpoint, and explains their statistical background. The chapter by Frellsen et al. overlaps slightly with the previous chapter, but has a more statistical slant. Knowledge based potentials can be formally understood in a Bayesian framework, which justifies, clarifies and extends them. The chapter by Frellsen et al. discusses the recently introduced reference ratio method, and highlights its theoretical and conceptual importance for one of the holy grails of structural bioinformatics: a rigorous and efficient probabilistic model of protein structure. Finally, the chapter by Podtelezhnikov and Wild discusses the application of another well-founded machine learning method to the construction of knowledge based potentials, namely contrastive divergence learning. The preliminary results reviewed in this chapter establish the method as promising for the future.

In Part III, we turn to directional statistics. Directional statistics concerns data on unusual manifolds such as the torus, the sphere or the real projective plane, which can be considered as a sphere with its antipodes identified. Examples of data from such manifolds include wind directions and dihedral angles in molecules. The need for directional statics can be understood by considering the fact that biomolecular structure is often expressed in terms of angles, and that the average of, for example, $1^{\circ}$ and $359^{\circ}$ is not 180 but zero. This is of course due to the fact that such data is naturally represented on the circle, rather than on the line. Hence, directional statistics is becoming vital for the formulation of probabilistic models of biomolecular structure. The two chapters by Mardia and Frellsen and by Kent are of a more technical nature and provide information on parameter estimation and sampling for two distributions from directional statistics that are of particular relevance for biomolecular structure. Kent's chapter discusses the Fisher-Bingham 5 (or Kent) distribution, which can be used to model data on the two-dimensional sphere, that is, data consisting of unit vectors. Frellsen and Mardia discuss the univariate, bivariate and multivariate von Mises distributions, for data on the circle and the torus, respectively. The latter case is of special relevance for modeling the $\phi$ and $\psi$ angles in proteins, which are well known from the celebrated Ramachandran plot.

Part IV explores the use of shape theory in comparing protein structures. Comparing and superimposing protein structures is one of the classic problems in structural bioinformatics. The chapter by Theobald discusses the superposition of proteins when their equivalent amino acids are known. Classically, this is done using a least squares criterion, but this leads to poor performance in many cases. Theobald describes a maximum likelihood alternative to the classic method, and also discusses a fully Bayesian extension. The problem of superimposing protein structures when 
the equivalent amino acids are not known is subsequently discussed in the chapter by Mardia and Nyirongo. They review a Bayesian model that is built upon a Poisson process and a proper treatment of the prior distributions of the nuisance variables.

Part V is concerned with the use of graphical models in structural bioinformatics. The chapter by Boomsma et al. introduces probabilistic models of RNA and protein structure that are based on the happy marriage of directionals statistics and dynamic Bayesian networks. These models can be used in conformational sampling, but are also vital elements for the formulation of a complete probabilistic description of protein structure. Yanover and Fromer discuss belief propagation in graphical models to solve the classic problem of side chain placement on a given protein main chain, which is a key problem in protein design.

In the sixth, final part, the inference of biomolecular structure from experimental data is discussed. This is of course one of the most fundamental applications of statistics in structural biology and structural bioinformatics. It is telling that currently most structure determination methods rely on a so-called pseudo-energy, which combines a physical force field with a heuristic force field that brings in the effect of the experimental data. Only recently methods have emerged that formulate this problem of inference in a rigorous, Bayesian framework. Habeck discusses Bayesian inference of protein structure from NMR data, while Hansen discusses the case of SAXS.

Many of the concepts and methods presented in the book are novel, and have neither been tested, honed or proven in large scale applications. However, the editors have little doubt that many concepts presented in this book will have a profound effect on the incremental solution of one of the great challenges in science today. 


\section{Acknowledgements}

We thank Justinas V. Daugmaudis for his invaluable help with typesetting the book in IATEX. In addition, we thank Christian Andreetta, Joe Herman, Kresten LindorffLarsen, Simon Olsson and Jan Valentin for comments and discussion.

This book was supported by the Danish Research Council for Technology and Production Sciences (FTP), under the project "Protein structure ensembles from mathematical models - with application to Parkinson's $\alpha$-synuclein", and the Danish Research Council for Strategic Research (NaBiIT), under the project "Simulating proteins on a millisecond time-scale". 



\section{Contents}

\section{Part I Foundations}

1 An Overview of Bayesian Inference and Graphical Models .......... 3 Thomas Hamelryck

2 Monte Carlo Methods for Inference in High-Dimensional Systems Jesper Ferkinghoff-Borg

\section{Part II Energy Functions for Protein Structure Prediction}

3 On the Physical Relevance and Statistical Interpretation of Knowledge-Based Potentials

Mikael Borg, Thomas Hamelryck, and Jesper FerkinghoffBorg

4 Towards a General Probabilistic Model of Protein Structure: The Reference Ratio Method Jes Frellsen, Kanti V. Mardia, Mikael Borg, Jesper Ferkinghoff-Borg, and Thomas Hamelryck

5 Inferring Knowledge Based Potentials Using Contrastive Divergence

Alexei A. Podtelezhnikov and David L. Wild

Part III Directional Statistics for Biomolecular Structure

6 Statistics of Bivariate von Mises Distributions Kanti V. Mardia and Jes Frellsen

7 Statistical Modelling and Simulation Using the Fisher-Bingham Distribution John T. Kent 


\section{Part IV Shape Theory for Protein Structure Superposition}

8 Likelihood and Empirical Bayes Superposition of Multiple Macromolecular Structures

Douglas L. Theobald

9 Bayesian Hierarchical Alignment Methods Kanti V. Mardia and Vysaul B. Nyirongo

\section{Part V Graphical Models for Structure Prediction}

10 Probabilistic Models of Local Biomolecular Structure and Their Applications

Wouter Boomsma, Jes Frellsen, and Thomas Hamelryck

11 Prediction of Low Energy Protein Side Chain Configurations Using Markov Random Fields

Chen Yanover and Menachem Fromer

Part VI Inferring Structure from Experimental Data

12 Inferential Structure Determination from NMR Data Michael Habeck

13 Bayesian Methods in SAXS and SANS Structure Determination..... 313 Steen Hansen

References

Index 


\section{Contributors}

Wouter Boomsma Department of Astronomy and Theoretical Physics, Lund University, Lund, Sweden, wouter@thep.lu.se

Department of Biomedical Engineering, DTU Elektro, Technical University of Denmark, Lyngby, Denmark, wb@elektro.dtu.dk

Mikael Borg The Bioinformatics Centre, Department of Biology, University of Copenhagen, Ole Maaløesvej 5, 2200 Copenhagen, Denmark, borg@binf.ku.dk

Jesper Ferkinghoff-Borg Department of Biomedical Engineering, DTU Elektro, Technical University of Denmark, Lyngby, Denmark, jfb@elektro.dtu.dk

Jes Frellsen The Bioinformatics Centre, Department of Biology, University of Copenhagen, Ole Maaløesvej 5, 2200 Copenhagen, Denmark, frellsen@binf.ku.dk

Menachem Fromer The Hebrew University of Jerusalem, Jerusalem, Israel, fromer@cs.huji.ac.il

Michael Habeck Department of Protein Evolution, Max-Planck-Institute for Developmental Biology, Spemannstr. 35, Tübingen, Germany, michael.habeck@ tuebingen.mpg.de

Department of Empirical Inference, Max-Planck-Institute for Intelligent Systems, Spemannstr. 38, Tuebingen, Germany

Thomas Hamelryck The Bioinformatics Centre, Department of Biology, University of Copenhagen, Ole Maaløesvej 5, 2200 Copenhagen, Denmark, thamelry@ binf.ku.dk

Steen Hansen Department of Basic Sciences and Environment, University of Copenhagen, Faculty of Life Sciences, Thorvaldsensvej 40, DK-1871 FRB C, Frederiksberg, Denmark, slh@life.ku.dk

John T. Kent Department of Statistics, University of Leeds, Leeds LS2 9JT, UK 
Kanti V. Mardia Department of Statistics, University of Leeds, Leeds LS2 9JT, UK, k.v.mardia@leeds.ac.uk

Vysaul B. Nyirongo Statistics Division, United Nations, New York, NY 10017, USA, nyirongov@un.org

Alexei A. Podtelezhnikov Michigan Technological University, Houghton, MI, USA, apodtele@gmail.com

Douglas L. Theobald Brandeis University, 415 South St, Waltham MA, USA, dtheobald@brandeis.edu

David L. Wild University of Warwick, Coventry, CV4 7AL, UK, d.1.wild@ warwick.ac.uk

Chen Yanover Fred Hutchinson Cancer Research Center, Seattle, WA, USA, cyanover@fhcrc.org 


\section{Acronyms}

$\begin{array}{ll}\text { AIC } & \text { Akaike information criterion } \\ \text { BIC } & \text { Bayesian information criterion } \\ \text { BMMF } & \text { Best max-marginal first } \\ \text { BN } & \text { Bayesian network } \\ \text { BP } & \text { Belief propagation } \\ \text { CASP } & \text { Critical Assessment of Techniques for Protein Structure Prediction } \\ \text { CD } & \text { Contrastive divergence } \\ \text { CPT } & \text { Conditional probability table } \\ \text { DBN } & \text { Dynamic Bayesian network } \\ \text { DEE } & \text { Dead end elimination } \\ \text { DIC } & \text { Deviance information criterion } \\ \text { EM } & \text { Expectation maximization } \\ \text { GA } & \text { Genetic algorithm } \\ \text { GBP } & \text { Generalized belief propagation } \\ \text { GE } & \text { Generalized ensemble } \\ \text { GMEC } & \text { Global minimum energy configuration } \\ \text { GMH } & \text { Generalized multihistogram equations } \\ \text { GIFT } & \text { Generalized indirect Fourier transformation } \\ \text { HMM } & \text { Hidden Markov model } \\ \text { IFT } & \text { Indirect Fourier transformation } \\ \text { ILP } & \text { Integer linear programming } \\ \text { ISD } & \text { Inferential structure determination } \\ \text { JT } & \text { Junction tree } \\ \text { KBP } & \text { Knowledge based potential } \\ \text { LP } & \text { Linear programming } \\ \text { MAP } & \text { Maximum a posteriori } \\ \text { MCMC } & \text { Markov chain Monte Carlo } \\ \text { MCSA } & \text { Monte Carlo simulated annealing } \\ \text { ML } & \text { Maximum likelihood } \\ \text { MM } & \text { Max-marginal } \\ \text { MPLP } & \text { Max-product linear programming } \\ & \end{array}$




$\begin{array}{ll}\text { MRF } & \text { Markov random field } \\ \text { MUCA } & \text { Multicanonical ensemble } \\ \text { NMR } & \text { Nuclear magnetic resonance spectroscopy } \\ \text { OLS } & \text { Ordinary least squares } \\ \text { PDB } & \text { Protein data bank } \\ \text { PDF } & \text { Probability density function } \\ \text { PMF } & \text { Potentials of mean force } \\ \text { REMD } & \text { Replica-exchange molecular dynamics } \\ \text { RMSD } & \text { Root mean square deviation } \\ \text { SANS } & \text { Small angle neutron scattering } \\ \text { SAS } & \text { Small angle scattering } \\ \text { SAXS } & \text { Small angle X-ray scattering } \\ \text { SCMF } & \text { Self-consistent mean field } \\ \text { SCWRL } & \text { Side-chains with a rotamer library } \\ \text { SLS } & \text { Static light scattering } \\ \text { SPRINT } & \text { Side-chain prediction inference toolbox } \\ \text { STRIPES } & \text { Spanning tree inequalities and partitioning for enumerating } \\ & \text { Solutions } \\ \text { TRMP } & \text { Tree-reweighted max-product } \\ \text { tBMMF } & \text { Type-specific BMMF } \\ \text { WHAM } & \text { Weighted histogram analysis method } \\ \text { WL algorithm } & \text { Wang-Landau algorithm } \\ & \end{array}$

